\title{
Ultralocal Scalar Field Models
}

\author{
JOHN R. KLAUDER \\ Bell Telephone Laboratories, Incorporated, Murray Hill, New Jersey
}

Received March 9, 1970

\begin{abstract}
In this paper the quantum theory of ultralocal scalar fields is developed. Such fields are distinguished by the independent temporal development of the field at each spacial point. Although the classical theories fit into the canonical framework, this is not the case for the quantum theories (with the exception of the free field). Explicit operator constructions are given for the field and the Hamiltonian as well as several other operators, and the calculation of the truncated vacuum expectation values is reduced to an associated single degree of freedom calculation. It is shown that construction of the Hamiltonian from the field, as well as the transition from the interacting to the noninteracting theories entails various infinite renormalizations which are made explicit.
\end{abstract}

\section{Introduction}

In this paper we consider the quantum theory of scalar field models for which the classical Hamiltonian reads

$$
H=\int\left\{\frac{1}{2} \pi_{c l}^{2}(\boldsymbol{x})+\frac{1}{2} m_{0}^{2} \varphi_{c l}^{2}(\boldsymbol{x})+V\left[\varphi_{c l}(\boldsymbol{x})\right]\right\} d \boldsymbol{x} .
$$

Here $\pi_{c l}(\boldsymbol{x})$ and $\varphi_{c l}(\boldsymbol{x})$ denote the classical momentum and field, respectively, and $\boldsymbol{x}$ is a point in configuration space (of arbitrary dimension). Such a theory is ultralocal - differing from a relativistic theory by the absence of the term $\frac{1}{2}\left[\nabla \varphi_{c l}(x)\right]^{2}$ - and it is distinguished by the independent evolution of the dynamics at each point of space ${ }^{1}$. This feature, at once the key to their solubility, is likewise responsible for the extremely delicate nature of these models.

The quantum theory of such models does not fit into the canonical framework whenever there is nontrivial interaction due to the lack of canonical commutation relations. There is a field operator $\varphi(x)$ (requiring a spacial smearing function alone), and a Hamiltonian $\mathscr{H}$, and consequently there exists a time-dependent field

$$
\varphi(\boldsymbol{x}, t)=e^{i \mathscr{H} t} \varphi(\boldsymbol{x}) e^{-i \mathscr{H} t} .
$$

However, there is no $\dot{\varphi}(\boldsymbol{x})=i[\mathscr{H}, \varphi(\boldsymbol{x})]$ operator. The domains of the operators $\varphi(x)$ and $\mathscr{H}$ just do not permit $\dot{\varphi}(\boldsymbol{x})$ to be defined. The simple

\footnotetext{
${ }^{1}$ A number of studies of these models have appeared previously [1].
} 
operator solution we present in Sec. 2 makes these properties quite clear. This solution is justified in Sec. 3, along with a discussion of the transition to the noninteracting theory.

For clarity and since the construction of these models is comparatively simple, we adopt a physical style of presentation. Rigorous demonstrations may be formulated in a straight-forward fashion from well-known properties of the usual Fock operators.

\section{Construction of the Operator Solutions}

We begin our construction of the quantum theory by introducing a family of annihilation and creation operators $A(x, \lambda)$ and $A^{\dagger}(x, \lambda)$ defined over configuration space and for $-\infty<\lambda<\infty$. They fulfill the standard commutation relation

$$
\left[A(\boldsymbol{x}, \lambda), A^{\dagger}\left(\boldsymbol{x}^{\prime}, \lambda^{\prime}\right)\right]=\delta\left(\boldsymbol{x}-\boldsymbol{x}^{\prime}\right) \delta\left(\lambda-\lambda^{\prime}\right),
$$

and we introduce a ground state $|0\rangle$ for which

$$
A(\boldsymbol{x}, \lambda)|0\rangle=0 .
$$

Stating that there is only one vector annihilated by all the $A(x, \lambda)$, we clinch the fact that the $A$ operators have the usual Fock representation. We let $c(\lambda)$ denote a real, even, nowherevanishing function of $\lambda$, and we define the translated Fock operator

$$
B(\boldsymbol{x}, \lambda)=A(\boldsymbol{x}, \lambda)+c(\lambda),
$$

which obviously obeys the relation

$$
\left[B(\boldsymbol{x}, \lambda), B^{\dagger}\left(\boldsymbol{x}^{\prime}, \lambda^{\prime}\right)\right]=\delta\left(\boldsymbol{x}-\boldsymbol{x}^{\prime}\right) \delta\left(\lambda-\lambda^{\prime}\right) .
$$

The function $c(\lambda)$ is not chosen square integrable, but instead we assume that

$$
\int\left(\frac{|\lambda|}{1+|\lambda|}\right) c^{2}(\lambda) d \lambda<\infty
$$

Indeed the ill behavior of $c(\lambda)$ need only occur at $\lambda=0$, and we will assume for simplicity that all moments of $c^{2}(\lambda)$ exist. As a suitable example it suffices to consider

$$
c(\lambda)=C|\lambda|^{-\frac{1}{2}} e^{-\frac{1}{2} a \lambda^{2}-\frac{1}{4} b \lambda^{4}} .
$$

The field operator for the models in question is given by

$$
\varphi(\boldsymbol{x}) \equiv \int B^{\dagger}(\boldsymbol{x}, \lambda) \lambda B(\boldsymbol{x}, \lambda) d \lambda,
$$


which is self-adjoint when smeared with real test functions $f(\boldsymbol{x})$. If we set

$$
\varphi(f) \equiv \int \varphi(\boldsymbol{x}) f(\boldsymbol{x}) d \boldsymbol{x},
$$

then the expectation functional

$$
\begin{aligned}
E(f) & \equiv\left\langle 0\left|e^{i \psi(f)}\right| 0\right\rangle \\
& =\exp \left\{-\int d \boldsymbol{x} \int\left[1-e^{i \lambda f(\boldsymbol{x})}\right] c^{2}(\lambda) d \lambda\right\}
\end{aligned}
$$

as follows from standard properties of creation and annihilation operators [2]. The nonsquare integrability of $c(\lambda)$ ensures that $\varphi(f)$ has no point spectrum $[3,2]$ and specifically that it does not annihilate any state vector which is evidently undesirable on physical grounds ${ }^{2}$.

The form of the integral over configuration space in the exponent reflects the statistical independence of the field at different space points. This property is in no way lost when dynamical evolution is included, and is incorporated in our definition of $\mathscr{H}$. We define the Hamiltonian by

$$
\begin{aligned}
\mathscr{H} & =\int d \boldsymbol{x} \int B^{\dagger}(\boldsymbol{x}, \lambda)\left\{-\frac{1}{2} \frac{\partial^{2}}{\partial \lambda^{2}}+v(\lambda)\right\} B(\boldsymbol{x}, \lambda) d \lambda, \\
& =\int d \boldsymbol{x} \int A^{\dagger}(\boldsymbol{x}, \lambda)\left\{-\frac{1}{2} \frac{\partial^{2}}{\partial \lambda^{2}}+v(\lambda)\right\} A(\boldsymbol{x}, \lambda) d \lambda .
\end{aligned}
$$

Here, to obtain the second equation we have linked $v(\lambda)$ and $c(\lambda)$ such that

$$
v(\lambda) \equiv \frac{1}{2} c^{\prime \prime}(\lambda) / c(\lambda) .
$$

That is, the dynamics and the field representation are linked. The equality of the two expressions for $\mathscr{H}$ is seen to be more plausible when it is recognized that

$$
h \equiv-\frac{1}{2} \frac{\partial^{2}}{\partial \hat{\lambda}^{2}}+v(\lambda) \equiv b^{\dagger} b,
$$

where

$$
b \equiv \frac{1}{\sqrt{2}} c(\lambda) \frac{\partial}{\partial \lambda} c(\lambda)^{-1} .
$$

With $h>0$ it is clear that $\mathscr{H} \geqq 0$. The state $|0\rangle$ is in fact the only state with eigenvalue zero.

The canonical appearance of the field and Hamiltonian in the " $\lambda$ space" is not accidental. Indeed, $\dot{\lambda} \equiv-i[\lambda, h]=(-i \partial / \partial \lambda)$ generates the conjugate operator to $\lambda$, as with any respectable canonical theory. However,

${ }^{2}$ In the model of Streater (Ref. [1]) the field and momentum have "mixed" spectrum, annihilate a particular state and do not fulfill canonical commutation relations. 
in the field case

$$
\begin{aligned}
\dot{\varphi}(\boldsymbol{x}) & \equiv-i[\varphi(\boldsymbol{x}), \mathscr{H}] \\
& =-i \int B^{\dagger}(\boldsymbol{x}, \lambda)[\lambda, h] B(\boldsymbol{x}, \lambda) d \lambda \\
& =-i \int B^{\dagger}(\boldsymbol{x}, \lambda)(\partial / \partial \lambda) B(\boldsymbol{x}, \lambda) d \lambda
\end{aligned}
$$

which is not an operator due to our assumptions regarding $c(\lambda)$. Furthermore,

$$
\left[\varphi(\boldsymbol{x}), \dot{\varphi}\left(\boldsymbol{x}^{\prime}\right)\right]=i \delta\left(\boldsymbol{x}-\boldsymbol{x}^{\prime}\right) \int B^{\dagger}(\boldsymbol{x}, \lambda) B(\boldsymbol{x}, \lambda) d \lambda
$$

which is also not defined. Evidently it is the $c$-number term which diverges here, being given by

$$
i \delta\left(\boldsymbol{x}-\boldsymbol{x}^{\prime}\right) \int c^{2}(\lambda) d \lambda .
$$

The Heisenberg field operator is, however, well defined. It follows that

$$
\begin{aligned}
\varphi(\boldsymbol{x}, t) & \equiv e^{i \mathscr{H} t} \varphi(\boldsymbol{x}) e^{-i \mathscr{H} t} \\
& =\int B^{\dagger}(\boldsymbol{x}, \lambda) e^{i \hbar t} \lambda e^{-i \hbar t} B(\boldsymbol{x}, \lambda) d \lambda \\
& \equiv \int B^{\dagger}(\boldsymbol{x}, \lambda) \lambda(t) B(\boldsymbol{x}, \lambda) d \lambda .
\end{aligned}
$$

The well-defined nature of this expression hinges on the condition that $(p=1,2)$

$$
\int|\lambda|^{p}\left|e^{-i \hbar t} c(\lambda)\right|^{2} d \lambda<\infty
$$

which is evidently true since our "linking" yields

$$
e^{-i \hbar t} c(\lambda)=c(\lambda)
$$

and we have already assumed that

$$
\int|\lambda|^{p} c^{2}(\lambda) d \lambda<\infty
$$

Let us consider the expression

$$
\begin{aligned}
e^{-i \psi(f)} \mathscr{H} e^{i \psi(f)}= & \int d \boldsymbol{x} \int B^{\dagger}(\boldsymbol{x}, \lambda) e^{-i \lambda f(\boldsymbol{x})} h e^{i \lambda f(\boldsymbol{x})} B(\boldsymbol{x}, \lambda) d \lambda \\
= & \int d \boldsymbol{x} \int B^{\dagger}(\boldsymbol{x}, \lambda)\left\{-\frac{1}{2}\left[\frac{\partial}{\partial \lambda}+i f(\boldsymbol{x})\right]^{2}+v(\lambda)\right\} \\
& \times B(\boldsymbol{x}, \lambda) d \lambda .
\end{aligned}
$$

The ground state $|0\rangle$ is not in the domain of this operator (unless $f \equiv 0$ ), and one even finds that

$$
\left\langle 0\left|e^{-i \psi(f)} \mathscr{H} e^{i \psi(f)}\right| 0\right\rangle=\frac{1}{2} \int f^{2}(\boldsymbol{x}) d \boldsymbol{x} \int c^{2}(\lambda) d \lambda=\infty .
$$


Such a property invalidates the relation of Araki yielding the matrix elements of the Hamiltonian in such states [4]. In addition, the weak correspondence principle is not available to us to determine a suitable form for the associated classical Hamiltonian [5].

Various vacuum expectation values may be reexpressed in terms of " $\lambda$-space" calculations. Consider, for example, the two-point function which becomes

$$
\begin{aligned}
\left\langle 0\left|\varphi(\boldsymbol{x}, t) \varphi\left(\boldsymbol{x}^{\prime}\right)\right| 0\right\rangle & =\left\langle 0\left|\varphi(\boldsymbol{x}) e^{-i \mathscr{H} t} \varphi\left(\boldsymbol{x}^{\prime}\right)\right| 0\right\rangle \\
& =\delta\left(\boldsymbol{x}-\boldsymbol{x}^{\prime}\right) \int c(\lambda) \lambda e^{-i \hbar t} \lambda c(\lambda) d \lambda,
\end{aligned}
$$

where use has been made of the fact that

$$
\left\langle 0\left|\varphi\left(\boldsymbol{x}^{\prime}\right)\right| 0\right\rangle=\int \lambda c^{2}(\lambda) d \lambda=0 .
$$

The analog of the spectral weight function $\varrho(\omega)$ is found from the relation

$$
\begin{aligned}
-i \Delta_{+}^{\prime}(t) & \equiv \int c(\lambda) \lambda e^{-i \hbar t} \lambda c(\lambda) d \lambda \\
& \equiv \frac{1}{2} \int_{0}^{\infty} e^{-i \omega t} \varrho(\omega) d \omega / \omega .
\end{aligned}
$$

Evidently

$$
\frac{1}{2} \int_{0}^{\infty} \varrho(\omega) d \omega / \omega=\int \lambda^{2} c^{2}(\lambda) d \lambda<\infty,
$$

while

$$
\int_{0}^{\infty} \varrho(\omega) d \omega=\int c^{2}(\lambda) d \lambda=\infty .
$$

The detailed high energy behavior of $\varrho(\omega)$ depends on the specific model, i.e., on $c(\lambda)$.

More generally, the $n$-point truncated vacuum expectation values are given by

$$
\begin{aligned}
&\left\langle 0\left|\varphi\left(\boldsymbol{x}_{1}, t_{1}\right) \varphi\left(\boldsymbol{x}_{2}, t_{2}\right) \ldots \varphi\left(\boldsymbol{x}_{n}, t_{n}\right)\right| 0\right\rangle^{T} \\
&= \delta\left(\boldsymbol{x}_{1}-\boldsymbol{x}_{2}\right) \delta\left(\boldsymbol{x}_{2}-\boldsymbol{x}_{3}\right) \ldots \delta\left(\boldsymbol{x}_{n-1}-\boldsymbol{x}_{n}\right) \\
& \times \int c(\lambda) \lambda\left(t_{1}\right) \lambda\left(t_{2}\right) \ldots \lambda\left(t_{n}\right) c(\lambda) d \lambda .
\end{aligned}
$$

Again the details of the multi-time behavior depend on the specific choice of $c(\lambda)$.

Evidently the function $c(\lambda)$ characterizes the theory and determines all its physical properties. Two functions $c(\lambda)$ which differ by a proportionality factor lead to the same definition of $h$ and consequently the same spectrum for $\mathscr{H}$ (cf., end of Sec. 3). We note also that distinct $c(\lambda)$ 
lead to unitarily inequivalent representations for $\varphi(x)$, representations which may not even be unitarily equivalent in compact regions of configuration space [2].

Another operator familiar in field problems is the space-translation generator $\mathscr{P}$. This operator is characterized by the property that

$$
[\mathscr{P}, \varphi(\boldsymbol{x})]=-i \nabla \varphi(\boldsymbol{x})
$$

with a similar relation holding for other local operators. In the present models this operator has the representation

$$
\mathscr{P}=-i \int d \boldsymbol{x} \int A^{\dagger}(\boldsymbol{x}, \lambda) \nabla A(\boldsymbol{x}, \lambda) d \lambda
$$

not unlike that in free theories apart from the additional $\lambda$ integration. Evidently, $[\mathscr{P}, \mathscr{H}]=0$ as is appropriate for a Euclidean invariant theory. In addition, $\mathscr{P}|0\rangle=0$, and $|0\rangle$ is nondegenerate for a noncompact configuration space.

\section{Justification of Assumptions, and Passage to the Free Field Limit}

By the very nature of the ultralocal feature of the models under study it is clear that the expectation functional $E(f)$ necessarily has the form

$$
E(f) \equiv\left\langle 0\left|e^{i \psi(f)}\right| 0\right\rangle=\exp \left\{-\int d \boldsymbol{x} L^{\prime}[f(\boldsymbol{x})]\right\},
$$

and thus is the characteristic functional of an infinitely-divisible stochastic variable [5]. With the added symmetry postulate $E(-f)=E(f)$ it follows that the most general form for $L^{\prime}$ is given by

$$
L^{\prime}[f]=\int(1-\cos \lambda f) \cdot d \sigma(\lambda)
$$

where $\sigma$ is odd [6]. Finiteness of $L^{\prime}$ requires only that

$$
\int\left(\frac{\lambda^{2}}{1+\lambda^{2}}\right) d \sigma(\lambda)<\infty .
$$

For $\varphi(f)$ to have only an absolutely continuous $(a c)$ spectrum it is sufficient if either $\sigma$ has a discontinuity at $\lambda=0$ or $\int d \sigma_{a c}(\lambda)=\infty$, or both [3]. Formally, the discontinuity is such that $d \sigma / d \lambda=(2 \mu)^{-1} \lambda^{-2} \delta(\lambda)$, where $\mu$ is a factor of proportionality. We thus accept this possibility and set

$$
\begin{aligned}
L^{\prime}[f] & =\frac{1}{4} \mu^{-1} f^{2}+\int(1-\cos \lambda f) c^{2}(\lambda) d \lambda \\
& \equiv L_{F}[f]+L[f],
\end{aligned}
$$


where, as indicated, the second contribution is chosen with an absolutely continuous $\sigma$. The first term $L_{F}[f]$ generates the expectation functional of the ultralocal, free Fock field $\varphi_{F}(\boldsymbol{x})$ of mass $\mu$. Indeed the complete field is the sum of two independent fields,

$$
\varphi^{\prime}(\boldsymbol{x})=\varphi_{F}(\boldsymbol{x})+\varphi(\boldsymbol{x}),
$$

which has the representation [2]

$$
\varphi^{\prime}(\boldsymbol{x})=\frac{1}{\sqrt{2 \mu}}\left[A_{F}(\boldsymbol{x})+A_{F}^{\dagger}(\boldsymbol{x})\right]+\int B^{\dagger}(\boldsymbol{x}, \lambda) \lambda B(\boldsymbol{x}, \lambda) d \lambda .
$$

The ultralocal character of the dynamics requires that the appropriate Hamiltonian $\mathscr{H}^{\prime}$ be bilinear in the creation and annihilation operators, and with any respect for the canonical theory $\mathscr{H}^{\prime}$ takes the form given by

$$
\mathscr{H}^{\prime}=\mu \int d \boldsymbol{x} A_{F}^{\dagger}(\boldsymbol{x}) A_{F}(\boldsymbol{x})+\int d \boldsymbol{x} \int A^{\dagger}(\boldsymbol{x}, \lambda) h A(\boldsymbol{x}, \lambda) d \lambda .
$$

Hence the two components of $\varphi^{\prime}, \varphi_{F}$ and $\varphi$, evolve in time independently of each other and thus behave as completely independent, uncoupled fields. We reject such solutions and assume that $\sigma(\lambda)$ has no discontinuity at $\lambda=0$.

Even though we restrict $\sigma(\lambda)$ to be absolutely continuous we can still recover the free theory through suitable limiting operations. For instance, the characteristic functional for the field $\varphi(f)$ becomes that of the free $\varphi_{F}(f)$ if a sequence $c_{\eta}^{2}(\lambda)$ is taken such that, roughly speaking, as $\eta \rightarrow \infty$,

$$
\lambda^{2} c_{\eta}^{2}(\lambda) \rightarrow(2 \mu)^{-1} \delta(\lambda)
$$

In such a case higher moments tend to zero, e.g., $\lambda^{4} c_{\eta}^{2}(\lambda) \rightarrow 0$, etc., and

$$
L_{\eta}[f]=\int(1-\cos \lambda f) c_{\eta}^{2}(\lambda) d \lambda \rightarrow \frac{1}{4} \mu^{-1} f^{2} .
$$

Obviously a number of sequences have this property, one such being given by

$$
c_{\eta}^{2}(\lambda)=\eta^{3} c^{2}(\eta \lambda)
$$

for some suitable initial choice $c_{1}(\lambda) \equiv c(\lambda)$ with

$$
(2 \mu)^{-1} \equiv \int \lambda^{2} c^{2}(\lambda) d \lambda .
$$

In a crude manner of speaking, the sequence $c_{\eta}(\lambda)$ has the effect of collapsing $\lambda$-space from the whole real line, $-\infty<\lambda<\infty$, to the single point $\lambda=0$. Whereas any $L^{2}$ function of $\lambda$ was previously admitted, only multiples of the single "function" $\lambda c_{\infty}(\lambda)$ are ultimately permitted reflecting the one-dimensional nature of collapsed " $\lambda$-space" in the limit $\eta \rightarrow \infty$. This insight is useful in understanding the transition of the field 
operator $\varphi(x)$ to the free operator $\varphi_{F}(\boldsymbol{x})$. Consider the expression

$$
\begin{aligned}
\varphi(\boldsymbol{x})= & \int A^{\dagger}(\boldsymbol{x}, \lambda) \lambda A(\boldsymbol{x}, \lambda) d \lambda \\
& +\int A(\boldsymbol{x}, \lambda) \lambda c(\lambda) d \lambda+\int A^{\dagger}(\boldsymbol{x}, \lambda) \lambda c(\lambda) d \lambda \\
& +\int \lambda c^{2}(\lambda) d \lambda,
\end{aligned}
$$

which follows from $B(\boldsymbol{x}, \lambda)=A(\boldsymbol{x}, \lambda)+c(\lambda)$. The last term vanishes in virtue of our assumption that $c(-\lambda)=c(\lambda)$. In the limit $\eta \rightarrow \infty$, the first (quadratic) term effectively vanishes because $\lambda$ - indeed $|\lambda|$ - has a vanishing average in the only allowed $\lambda$ state, $\lambda c_{\infty}(\lambda)$. However, the two linear terms remain, and as $\eta \rightarrow \infty$,

$$
\varphi(x) \rightarrow \varphi_{F}(x)=\frac{1}{\sqrt{2 \mu}}\left[A_{F}(x)+A_{F}^{\dagger}(\boldsymbol{x})\right],
$$

where

$$
\int A(\boldsymbol{x}, \lambda) \lambda c_{\eta}(\lambda) d \lambda \rightarrow \frac{1}{\sqrt{2 \mu}} A_{F}(\boldsymbol{x}) .
$$

The analysis of the transition of the general Hamiltonian to the free field Hamiltonian is somewhat more delicate because of domain questions. The resultant Hamiltonian must be proportional to $\int A_{F}^{\ddagger}(\boldsymbol{x})$ $A_{F}(\boldsymbol{x}) d \boldsymbol{x}$ which does lead to a well-defined canonical operator $\dot{\varphi}_{F}(\boldsymbol{x})$ $=-i\left[\varphi_{F}(x), \mathscr{H}_{F}\right]$ with proper canonical commutation relations

$$
\left[\varphi_{F}(x), \dot{\varphi}_{F}\left(x^{\prime}\right)\right]=i \delta\left(x-x^{\prime}\right) .
$$

Now, the Hamiltonians we must consider all have the form

$$
\begin{aligned}
\mathscr{H} & =\int d \boldsymbol{x} \int A^{\dagger}(\boldsymbol{x}, \lambda) h A(\boldsymbol{x}, \lambda) d \lambda \\
& =\int d \boldsymbol{x} \int[b A(\boldsymbol{x}, \lambda)]^{\dagger}[b A(\boldsymbol{x}, \lambda)] d \lambda,
\end{aligned}
$$

where we have used the relation $h=b^{\dagger} b$. The expectation value of this expression in states of the form $\varphi(f)|0\rangle$ is infinite as we have previously noted, but to win canonical commutation rules we must make an infinite renormalization as well by the factor $\int c^{2}(\lambda) d \lambda$.

To incorporate these features let us consider the scaled and modified Hamiltonians

$$
\mathscr{H}_{\varepsilon} \equiv \frac{\int d \boldsymbol{x} \int_{|\varepsilon|}[b A(\boldsymbol{x}, \lambda)]^{\dagger}[b A(\boldsymbol{x}, \lambda)] d \lambda}{\int_{|\varepsilon|} c^{2}(\lambda) d \lambda},
$$

where

$$
\int_{|\varepsilon|} \equiv \int_{\varepsilon}^{\infty}+\int_{-\infty}^{-\varepsilon}
$$


excludes a portion of the $\lambda$ integration about $\lambda=0$. Note this exclusion occurs in the numerator as well as the denominator. We next evaluate the expectation of $\mathscr{H}_{\varepsilon}$ in a normalized state of the form $\varphi(f)|0\rangle$. The result becomes

$$
\begin{aligned}
\left\langle\mathscr{H}_{\varepsilon}\right\rangle & \equiv \frac{\left\langle 0\left|\varphi(f) \mathscr{H}_{\varepsilon} \varphi(f)\right| 0\right\rangle}{\left\langle 0\left|\varphi^{2}(f)\right| 0\right\rangle} \\
& =\frac{\frac{1}{2} \int f^{2}(\boldsymbol{x}) d \boldsymbol{x} \int_{|\varepsilon|}\left[c \frac{\partial}{\partial \lambda} c^{-1} \lambda c\right]^{2} d \lambda}{\int f^{2}(\boldsymbol{x}) d \boldsymbol{x} \int \lambda^{2} c^{2} d \lambda \int_{|\varepsilon|} c^{2} d \lambda}=\mu
\end{aligned}
$$

where we have used $(2 \mu)^{-1} \equiv \int \lambda^{2} c^{2} d \lambda$ and have noted the identical cancellation of the two $\varepsilon$-excluded integrals. Other $\lambda$-space states lead to vanishing expectation values as $\varepsilon \rightarrow 0$. For example, the function $\lambda^{2} c(\lambda)$ [rather than $\left.\lambda c(\lambda)\right]$ leads to the normalized mean value

$$
\left\langle\mathscr{H}_{\varepsilon}\right\rangle_{1}=\frac{\frac{1}{2} \int f^{2}(\boldsymbol{x}) d \boldsymbol{x} \int_{|\varepsilon|}\left[c \frac{\partial}{\partial \lambda} c^{-1} \lambda^{2} c\right]^{2} d \lambda}{\int f^{2}(\boldsymbol{x}) d \boldsymbol{x} \int \lambda^{4} c^{2} d \lambda \int_{|\varepsilon|} c^{2} d \lambda}
$$

which vanishes as $\varepsilon \rightarrow 0$ since the numerator remains finite. Consequently the limiting form of $\mathscr{H}_{\varepsilon}$ is sensitive to only the single state $\lambda c$; moreover, the mean $\left\langle\mathscr{H}_{\varepsilon}\right\rangle$ is independent of the shape of $c$ (depending only on the norm of $\lambda c$ ) which permits the limit $\eta \rightarrow \infty$ to be taken in the collapsing sequence $c_{\eta}^{2}(\lambda)=\eta^{3} c^{2}(\eta \lambda)$. With the scaling, modifying and limiting operations understood it follows that

$$
\mathscr{H} \rightarrow \mathscr{H}_{F} \equiv \mu \int A_{F}^{\dagger}(\boldsymbol{x}) A_{F}(\boldsymbol{x}) d \boldsymbol{x}
$$

the prefactor being uniquely - and correctly - given by the fact that $\mu=\left\langle\mathscr{H}_{\varepsilon}\right\rangle$.

Lastly consider the space-translation generator

$$
\mathscr{P}=-i \int d \boldsymbol{x} \int A^{\dagger}(\boldsymbol{x}, \lambda) \nabla A(\boldsymbol{x}, \lambda) d \lambda .
$$

To achieve the desired limiting form we need only note that the $\lambda$-space integration may be replaced with a sum over orthonormal states, the first of which is chosen as $\sqrt{2 \mu} \lambda c(\lambda)$. This first term in the expansion gives the desired expression $\mathscr{P}_{F}$ as $\eta \rightarrow \infty$, and all successive terms give vanishing contribution in the limit $\eta \rightarrow \infty$ due to the collapse of the $\lambda$ space. Consequently we have

$$
\mathscr{P} \rightarrow \mathscr{P}_{F}=-i \int d \boldsymbol{x} A_{F}^{\dagger}(\boldsymbol{x}) \nabla A_{F}(\boldsymbol{x}) .
$$

This completes our discussion of the transition from the interacting to the noninteracting theories. 
For the free theory the states $|f\rangle_{F}=\exp \left[i \varphi_{F}(f)\right]|0\rangle$ are coherent states for which

$$
A_{F}(x)|f\rangle_{F}=i(2 \mu)^{-\frac{1}{2}} f(\boldsymbol{x})|f\rangle_{F} .
$$

The two-point function

$$
\left\langle 0\left|\varphi_{F}(\boldsymbol{x}) e^{-i \mathscr{K}_{F} t} \varphi_{F}\left(\boldsymbol{x}^{\prime}\right)\right| 0\right\rangle=\delta\left(\boldsymbol{x}-\boldsymbol{x}^{\prime}\right)(2 \mu)^{-1} e^{-i \mu t}
$$

is the only nonvanishing truncated vacuum expectation value, which implies that the spectrum of $\mathscr{H}_{F}$ is $\{0, \mu, 2 \mu, \ldots\}$. It is noteworthy for the interacting models that the states $|f\rangle \equiv \exp [i \varphi(f)]|0\rangle$ are "coherent" in the sense that [2]

$$
A(\boldsymbol{x}, \lambda)|f\rangle=\left(e^{i \lambda f(\boldsymbol{x})}-1\right) c(\lambda)|f\rangle .
$$

The auxiliary field

$$
\Phi(x, \lambda)=\frac{1}{\sqrt{2}}\left[A(x, \lambda)+A^{\dagger}(x, \lambda)\right]
$$

is self-adjoint when smeared with a real test function $h(\boldsymbol{x}, \lambda)$, but it is unphysical because of the appearance of $\lambda$. It follows that the two-point function

$$
\left\langle 0\left|\Phi(\boldsymbol{x}, \lambda) e^{-i \mathscr{H} t} \Phi\left(\boldsymbol{x}^{\prime}, \lambda^{\prime}\right)\right| 0\right\rangle=\frac{1}{2} \delta\left(\boldsymbol{x}-\boldsymbol{x}^{\prime}\right)\left(\lambda\left|e^{-i \hbar t}\right| \lambda^{\prime}\right)
$$

where $\mid \lambda)$ denotes $\delta$-normalized eigenvectors in the $\lambda$-space. All other truncated vacuum expectation values for $\Phi(\boldsymbol{x}, \lambda, t)$ vanish, which makes the simple structure of the theory immediately evident. It follows, e.g., that the spectrum of $\mathscr{H}$ bears the same relation to that of $h$ as is the case in the free theory [2]. However, it is the field $\varphi(\boldsymbol{x}, t)$ which is the physical field and not $\Phi(\boldsymbol{x}, \lambda, t)$. Polynomials in $\varphi(\boldsymbol{x})$ (at fixed $t$ ) span the same Hilbert space when acting on the vacuum as polynomials in $\Phi(\boldsymbol{x}, \lambda)$. In addition, the algebra of operators formed from $\varphi(x, t),|t|<\varepsilon$, is complete (i.e., irreducible).

\section{Conclusion}

What theory has been quantized? To gain some perspective on this problem let us consider the class of examples in which

$$
c(\lambda)=|\lambda|^{-\frac{1}{2}} \exp [-y(\lambda)],
$$

where $y(\lambda)$ is an even, positive polynomial. Then it follows that

$$
v(\lambda)=\frac{1}{2}\left\{\frac{3}{4} \lambda^{-2}+\lambda^{-1} y^{\prime}(\lambda)+y^{\prime 2}(\lambda)-y^{\prime \prime}(\lambda)\right\} \equiv \frac{3}{8} \lambda^{-2}+v_{0}(\lambda) .
$$


Our conditions on $y(\lambda)$ ensure that

$$
v_{0}(\lambda)=\sum_{n=1}^{N} v_{2 n} \lambda^{2 n}
$$

an even polynomial with the constant term vanishing. We note that $v_{0}(\lambda)$ generates well-defined local operators; that is

$$
\mathscr{V}_{0}(\boldsymbol{x}) \equiv \int \boldsymbol{B}^{\dagger}(\boldsymbol{x}, \lambda) v_{0}(\lambda) B(\boldsymbol{x}, \lambda) d \lambda
$$

is a self-adjoint operator when smeared with a space-dependent test function. Consequently,

$$
k_{0} \equiv h-v_{0}(\lambda)=-\frac{1}{2} \frac{\partial^{2}}{\partial \lambda^{2}}+\frac{3}{8 \lambda^{2}}
$$

also generates a valid local operator, namely

$$
\mathscr{K}_{0}(\boldsymbol{x}) \equiv \int B^{\dagger}(\boldsymbol{x}, \lambda) k_{0} B(\boldsymbol{x}, \lambda) d \lambda .
$$

Note that neither of the last two terms making up $k_{0}$ generate, by themselves, valid local operators, but only in combination. The Hamiltonian is given by

$$
\mathscr{H}=\int\left[\mathscr{K}_{0}(\boldsymbol{x})+\mathscr{V}_{0}(\boldsymbol{x})\right] d \boldsymbol{x} .
$$

On these grounds it is not unreasonable to regard $\mathscr{V}_{0}(x)$ as the potential term and to regard $\mathscr{K}_{0}(\boldsymbol{x})$ as the regularized kinetic energy term.

In support of this interpretation we demonstrate that the potential $\mathscr{V}_{0}(\boldsymbol{x})$ can be constructed as a polynomial in the field operator $\varphi(\boldsymbol{x})$ in which infinite renormalizations are involved. To see this we first note that

$$
\varphi(\boldsymbol{x}) \varphi(\boldsymbol{y})=\delta(\boldsymbol{x}-\boldsymbol{y}) \int B^{\dagger}(\boldsymbol{x}, \lambda) \lambda^{2} B(\boldsymbol{x}, \lambda) d \lambda+! \varphi(\boldsymbol{x}) \varphi(\boldsymbol{y}) !
$$

where !! denotes normal ordering with respect to $B^{\dagger}$ and $B$. Thus a suitable and standard kind of limiting operation leads to

$$
Z \varphi^{2}(\boldsymbol{x}) \equiv \int B^{\dagger}(\boldsymbol{x}, \lambda) \lambda^{2} B(\boldsymbol{x}, \lambda) d \lambda \equiv \varphi_{r}^{2}(\boldsymbol{x})
$$

where formally $Z^{-1}=\delta(0)$. Observe that this renormalized multiplication effectively leads to multiplication in " $\lambda$-space". In like fashion

$$
Z^{2 n-1} \varphi^{2 n}(x) \equiv \int B^{\dagger}(x, \lambda) \lambda^{2 n} B(x, \lambda) d \lambda \equiv \varphi_{r}^{2 n}(x),
$$

which means that

$$
\mathscr{V}_{0}(\boldsymbol{x})=\sum_{n=1}^{N} v_{2 n} \varphi_{r}^{2 n}(\boldsymbol{x})=\sum_{n=1}^{N} v_{2 n} Z^{2 n-1} \varphi^{2 n}(\boldsymbol{x}) \equiv Z^{-1} v_{0}[Z \varphi(\boldsymbol{x})] .
$$


It is worth noting that choices of $c(\lambda)$ with different degrees of singularity are also permissible. In particular, $c(\lambda)$ may be chosen as

$$
c(\lambda)=|\lambda|^{-\gamma} \exp [-y(\lambda)],
$$

where $\gamma \geqq \frac{1}{2}$ [for $\varphi(f)$ to have an absolutely continuous spectrum], and $\gamma<\frac{3}{2}$ [for $\varphi(f)$ to be self-adjoint with a spacially-dependent test function]. In these cases the Hamiltonian operator can again be meaningfully divided into a potential term and a regularized kinetic energy term.

If $\gamma \geqq \frac{3}{2}$ the field $\varphi(\boldsymbol{x})$ does not become an operator after smearing with a spacially-dependent test function. Additional smearing over time may prove adequate and this can be ascertained by a study of the twopoint distribution

$$
-i \Delta_{+}^{\prime}(t)=\int c(\lambda) \lambda e^{-i \hbar t} \lambda c(\lambda) d \lambda .
$$

However, if space-time smearing makes $\varphi(\boldsymbol{x}, t)$ an operator one may also work with the auxiliary field

$$
\theta(\boldsymbol{x}) \equiv \varphi_{r}^{2 p+1}(\boldsymbol{x})=\int B^{\dagger}(\boldsymbol{x}, \lambda) \lambda^{2 p+1} B(\boldsymbol{x}, \lambda) d \lambda
$$

which for sufficiently large $p$ will reallow a purely space-dependent test function. In this case the truncated vacuum expectation values of the field $\theta(\boldsymbol{x}, t)$ may be adopted as the objects of basic interest, and the formalism of Sec. 2 applies with evident modifications.

\section{References}

1. Wentzel, G.: Helv. Phys. Acta 13, 269 (1940).

Schiff, L. I.: Phys. Rev. 92, 766 (1953).

Araki, H.: Princeton thesis, 1960, Chap. V.

Ezawa, H.: Commun. math. Phys. 8, 261 (1968); 9. 38 (1968).

Streater, R. F.: Lectures at 1968 Karpacz Winter School, Karpacz, Poland (to be published).

2. Klauder, J. R.: J. Math. Phys. 11, 609 (1970).

3. Tucker, H. G.: Pacific J. Math. 12, 1125 (1962).

4. Araki, H.: J. Math. Phys. 1, 492 (1960), Sec. 8.

5. Klauder, J. R.: J. Math. Phys. 8, 2392 (1967).

6. Gel'fand, I. M., Vilenkin, N. Ya.: Generalized Functions, Vol. 4, Applications of Harmonic Analysis, translated by A. Feinstein, Chap. III. New York: Academic Press 1964.

John R. Klauder

Bell Telephone Laboratories Inc.

Murray Hill, New Jersey 07974, USA. 